This item was submitted to Loughborough's Research Repository by the author.

Items in Figshare are protected by copyright, with all rights reserved, unless otherwise indicated.

\title{
A general map matching algorithm for transport telematics applications
}

PLEASE CITE THE PUBLISHED VERSION

PUBLISHER

(C) Springer-Verlag

VERSION

AM (Accepted Manuscript)

LICENCE

CC BY-NC-ND 4.0

\section{REPOSITORY RECORD}

Quddus, Mohammed A., Washington Y. Ochieng, Lin Zhao, and Robert B. Noland. 2019. "A General Map Matching Algorithm for Transport Telematics Applications”. figshare. https://hdl.handle.net/2134/5261. 
This item was submitted to Loughborough's Institutional Repository (https://dspace.lboro.ac.uk/) by the author and is made available under the following Creative Commons Licence conditions.

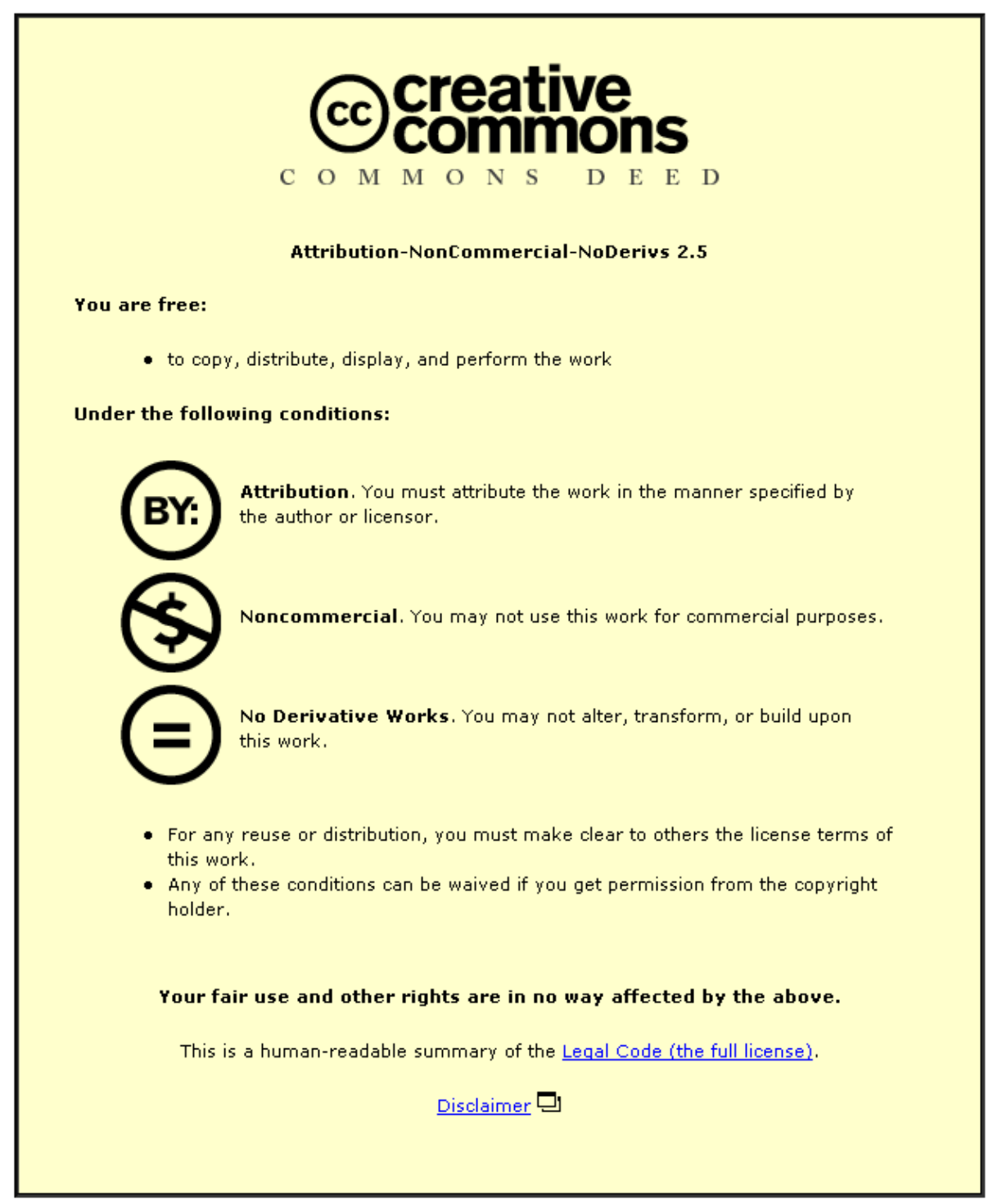

For the full text of this licence, please go to: http://creativecommons.org/licenses/by-nc-nd/2.5/ 


\title{
A general map matching algorithm for transport telematics applications
}

\author{
Mohammed A. Quddus \\ Washington Y. Ochieng \\ Lin Zhao \\ Robert B. Noland \\ Centre for Transport Studies \\ Dept. of Civil and Environmental Engineering \\ Imperial College London \\ London SW7 2AZ \\ Email: w.ochieng@imperial.ac.uk
}

\begin{abstract}
This paper describes a map-matching algorithm designed to support the navigational functions of a real-time vehicle performance and emissions monitoring system currently under development, and other transport telematics applications. The algorithm is used together with the outputs of an extended Kalman filter formulation for the integration of GPS and dead reckoning data, and a spatial digital database of the road network, to provide continuous, accurate and reliable vehicle location on a given road segment. This is irrespective of the constraints of the operational environment, thus alleviating outage and accuracy problems associated with the use of stand-alone location sensors. The map-matching algorithm has been tested using real field data and has been found to be superior to existing algorithms, particularly in how it performs at road intersections.
\end{abstract}

Key words: Transport telematics, GPS, map-matching, land vehicle navigation, optimal estimation. 


\section{$1 \quad$ INTRODUCTION}

Systems for monitoring and tracking vehicle movement offer many opportunities for the management of transport systems. The data collected from such systems also has the potential to provide a fuller understanding of the behavior of travelers and the consequences of that behavior both on the transport system and external effects, such as emissions of pollutants. This paper reports on a research and development project to create and demonstrate the capabilities of an accurate, reliable and cost effective real time data collection device, the vehicle performance and emissions monitoring system (VPEMS). The VPEMS will be fitted on vehicles to monitor vehicle and driver performance and the level of emissions and pollutant concentrations.

The navigation function of the VPEMS is responsible for the derivation of all spatial, temporal and spatio-temporal data of the vehicle including location in 3-dimensional space, time, slope, speed and acceleration. The required navigation performance (RNP) that needs to be achieved in built-up areas, where the impact of pollution from traffic is most serious, means that the navigation function cannot be supported by the global positioning system (GPS) alone. One possible solution is the integrated use of data from GPS with dead reckoning (DR) and map matching (MM). An extended Kalman filter (EKF) has been developed for the integration of GPS with dead reckoning sensor data (Zhao et al., 2002). This paper reports on the linking of the EKF with a new map matching algorithm to accurately locate a vehicle on a given road segment.

Positioning sensors such as GPS or even the integrated GPS/DR system employing EKF algorithms do not always provide the location of a vehicle on a given road segment with the required level of accuracy. This is due to the various error sources that affect such systems, such as orbital, clock, and propagation (Zhao et al., 2002). The availability of higher accuracy digital spatial road network data should make it possible to account for these errors and enable the actual vehicle position on a given road to be determined. This technique is known as map matching (MM). A formal definition of MM can be found in Bernstein and Kornhauser (1996), White et al. (2000) and Greenfeld (2002). The most complex algorithm is the general MM algorithm that does not assume any knowledge or any other information regarding the expected location of the vehicle. 
The objective of this paper is to describe the limitations of existing MM algorithms and describe a general algorithm, which is essential for the VPEMS project and other telematics applications. The proposed MM algorithm uses the output of the GPS/DR EKF algorithm. Its performance has been validated using data from a representative built-up environment (the Greater London area).

\section{EXISTING LITERATURE ON MAP MATCHING}

Map matching procedures vary from those using simple search techniques (Kim et al., 1996), to those using more complex mathematical techniques such as Kalman Filters (Tanaka et al., 1990). A number of different algorithms have been proposed for MM for different applications, each of which has advantages and disadvantages (Table 1). Some of these are reviewed below.

Bernstein and Kornhauser (1996) describe several algorithms (or parts of algorithms) for matching an estimated position to a network representation of the street system. Two things are apparent from this study. First, it is clear that this is a complex and fairly difficult task. Point-to-point and curve-to-point matching are unlikely to work very well, especially when there are errors in the position and/or errors in the network representation. Hence, other, more complicated algorithms must often be used. Second, though a number of different algorithms can be used, it seems clear that it is both important to perform some kind of curve-to-curve matching and to incorporate topological information in the algorithm. Bernstein and Kornhauser conclude that the more attention that is given to the topological information, the better the algorithm performs.

White et al. (2000) describe several algorithms (or parts of algorithms) for matching an estimated position to a network representation of the street system and attempt to evaluate four of them. Since most route changes occur at intersections, their study suggests that particular attention needs to be paid to the problems that arise at intersections. The discussion focuses on urban routes since most algorithms appear to work well on highways. However, they recommend that the algorithms need to be evaluated on a wider array of routes especially in urban areas. The limited number and variety of routes considered in their study preclude general conclusions from being made. 
Taylor et al. (2001) propose a novel method of map matching using differential GPS and height aiding, referred to as the road reduction filter $(R R F)$ algorithm. The general approach used is to improve the accuracy of the computed position of a vehicle via differential corrections and height aiding. The simple procedure used allows identification of all possible candidates for the correct road while systematically removing the wrong ones. The RRF algorithm is improved using road center-line network connectivity. The method was developed to deal with errors introduced by GPS selective availability (S/A) which was subsequently turned off in May 2000 (Ochieng and Sauer, 2001). Its benefits are still to be assessed in the S/A free environment.

Greenfeld (2002) reviews several approaches for solving the map matching problem and proposes a weighted topological algorithm. The algorithm is based on assessing the similarity between the characteristics of the street network and the positioning pattern of the user. The algorithm seems to work well even with relatively inferior or bouncy GPS data. Tests show that the procedure computes correct matches virtually everywhere. However, their study suggests that additional research is required to verify the accurate performance of the algorithm and to make an accurate position determination on a given road segment.

It is clear from the existing literature that a key component of any MM algorithm is to identify the correct link among the candidate links since one bad match can lead to a sequence of bad matches. The literature review has also revealed that particular attention has to be paid to topological aspects of the road network, matching processes at intersections and algorithm validation in complex route structure environments such as in built-up urban areas.

Table 1: Summary of literature

\begin{tabular}{|l|l|l|l|}
\hline Author (s), year & Algorithm & Procedures & Comments/Disadvantages \\
\hline $\begin{array}{l}\text { Bernstein and } \\
\text { Kornhauser (1996) } \\
\text { Kim et al (1996) } \\
\text { White et al (2000) }\end{array}$ & $\begin{array}{l}\text { Map matching as a } \\
\text { search problem or Point- } \\
\text { to-Point matching }\end{array}$ & $\begin{array}{l}\text { Map the GPS tick to the } \\
\text { 'closest' node or shape } \\
\text { point in the network }\end{array}$ & $\begin{array}{l}\text { a) only geometric information } \\
\text { b) never makes use of 'Historical' } \\
\text { information } \\
\text { c) very sensitive to the way in which } \\
\text { the network was digitized. That is, } \\
\text { other things being equal, arcs with } \\
\text { more shape points are more likely to } \\
\text { be properly matched }\end{array}$ \\
\hline
\end{tabular}




\begin{tabular}{|c|c|c|c|}
\hline $\begin{array}{l}\text { Bernstein and } \\
\text { Kornhauser (1996) } \\
\text { White et al. (2000) } \\
\text { Taylor et al. (2001) }\end{array}$ & Point-to-Curve matching & $\begin{array}{l}\text { Map the GPS tick to the } \\
\text { 'closest' arc in the } \\
\text { network (minimum } \\
\text { distance from the point to } \\
\text { the curve) }\end{array}$ & $\begin{array}{l}\text { a) only geometric information } \\
\text { b) lack of the use of historical } \\
\text { information and hence sometimes } \\
\text { assigns the incorrect link } \\
\text { c) quite unstable }\end{array}$ \\
\hline White et al. (2000) & $\begin{array}{l}\text { Point-to-Curve matching } \\
\text { with heading }\end{array}$ & $\begin{array}{l}\text { Same as Point-to-Curve } \\
\text { matching algorithm } \\
\text { except that it makes use } \\
\text { of the heading } \\
\text { information. If heading of } \\
\text { the current GPS trick is } \\
\text { not comparable to the } \\
\text { heading of the arc, then } \\
\text { the arc is discarded. }\end{array}$ & $\begin{array}{l}\text { a) better algorithm than point-to- } \\
\text { curve matching } \\
\text { b) GPS heading is sometimes very } \\
\text { inaccurate especially when the } \\
\text { vehicle speed is zero or very low. } \\
\text { Therefore, incorrect matching can } \\
\text { occur }\end{array}$ \\
\hline $\begin{array}{l}\text { Bernstein and } \\
\text { Kornhauser (1996) } \\
\text { White et al. (2000) } \\
\text { Taylor et al. (2001) }\end{array}$ & $\begin{array}{l}\text { Curve-to-Curve } \\
\text { matching }\end{array}$ & $\begin{array}{l}\text { Match arcs defined by a } \\
\text { series of GPS point } \\
\text { positions with arcs } \\
\text { defined by a set of points } \\
\text { that define partial road } \\
\text { center-lines. One method } \\
\text { used for matching two } \\
\text { curves (arcs) is to use the } \\
\text { distance between them. }\end{array}$ & $\begin{array}{l}\text { a) geometric information. } \\
\text { b) quite sensitive to outliers. } \\
\text { c) sometimes yield unexpected and } \\
\text { undesirable results }\end{array}$ \\
\hline $\begin{array}{l}\text { Bernstein and } \\
\text { Kornhauser (1996) } \\
\text { White et al. (2000) }\end{array}$ & $\begin{array}{l}\text { Improving Point-to- } \\
\text { Curve matching }\end{array}$ & $\begin{array}{l}\text { Given a known initial } \\
\text { point, the topology of the } \\
\text { network makes it possible } \\
\text { to reduce the set of likely } \\
\text { arcs dramatically }\end{array}$ & $\begin{array}{l}\text { Both geometric and topological } \\
\text { information are used. But quite } \\
\text { sensitive to the threshold that is used. } \\
\text { One bad match can lead to a } \\
\text { sequence of bad matches. }\end{array}$ \\
\hline $\begin{array}{l}\text { Bernstein and } \\
\text { Kornhauser (1996) } \\
\text { White et al. (2000) }\end{array}$ & $\begin{array}{l}\text { Improving Curve-to- } \\
\text { Curve matching }\end{array}$ & $\begin{array}{l}\text { Topological information } \\
\text { of the network is used } \\
\text { with curve-to-curve } \\
\text { matching }\end{array}$ & $\begin{array}{l}\text { Very complex to implement and a } \\
\text { real world experiment does not } \\
\text { consistently out perform other } \\
\text { algorithms. }\end{array}$ \\
\hline $\begin{array}{l}\text { Krakiwsky et al. } \\
(1988) \\
\text { Scott and Drane } \\
\text { (1994) } \\
\text { Jo et al. (1996) }\end{array}$ & $\begin{array}{l}\text { Map matching as a } \\
\text { statistical estimation }\end{array}$ & $\begin{array}{l}\text { In this approach, one } \\
\text { considers a sequence of } \\
\text { points and attempts to fit } \\
\text { a curve to them. This } \\
\text { curve is constrained to lie } \\
\text { on the network }\end{array}$ & $\begin{array}{l}\text { It is particularly elegant when the } \\
\text { model describing the 'physics of } \\
\text { motion' is simple e.g., movement is } \\
\text { only possible along a straight line. } \\
\text { Unfortunately, in most practical } \\
\text { applications, the physics of motion is } \\
\text { defined by the network. This makes it } \\
\text { quite difficult to model }\end{array}$ \\
\hline Greenfeld, J.S. (2002) & $\begin{array}{l}\text { Similarity criteria by } \\
\text { weighting system }\end{array}$ & $\begin{array}{l}\text { Topological analysis of } \\
\text { road network }\end{array}$ & $\begin{array}{l}\text { a) much more likely to be simple } \\
\text { and correct } \\
\text { b) but sometimes assigns incorrect } \\
\text { links especially at intersections } \\
\text { c) determination of vehicle position } \\
\text { is not robust }\end{array}$ \\
\hline
\end{tabular}




\section{SOME LIMITATIONS OF EXISTING MAP MATCHING ALGORITHMS}

Most of the limitations of the existing algorithms have been explained in White et al (2000) and Greenfeld (2002). However, other additional limitations that should be addressed can be easily identified. Examples of these are illustrated in Figures 1 and 2.

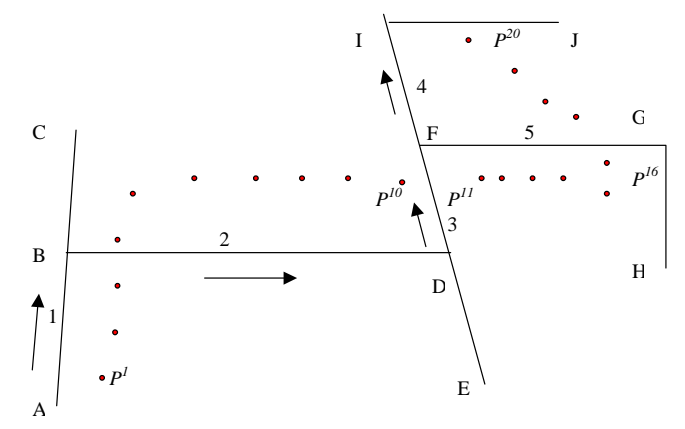

Figure 1: Incorrect match without the vehicle heading information at junction

In Figure 1, the points $P^{1}$ to $P^{20}$ represent the GPS/DR positions of a vehicle traveling on a sample network. It is relatively easy in this case to determine that the vehicle path is represented by the links 1-2-3-4. Applying the Greenfeld (2002) algorithm or any other algorithm explained in the above section, it is found that these points correctly match on the link until $P^{10}$. However, at point $P^{11}$, it selects link-5 as a correct link since the orientation of the GPS line between $P^{11}$ and $P^{12}$ is similar to link-5. The reason why the algorithm assigns an incorrect link is that it has not taken into account any heading information of the vehicle at $P^{11}$. There are many cases similar to this example. GPS/DR headings are fairly accurate and could be used to provide additional information in facilitating a correct identification of the link.

It is also possible to obtain GPS/DR positions as shown in Figure 2. Between points $P^{9}$ and $P^{27}$ the vehicle is stationary for about 18 seconds. The various points in between $P^{9}$ and $P^{27}$ reflect the error in GPS position determination. It is obvious that it would not be possible to identify the correct link either using the algorithm proposed by Greenfeld (2002) or any of the other map matching procedures described in the literature review section. One way to overcome this problem is to consider speed information from GPS/DR and assume a single position fix when the vehicle is stationary (i.e. zero speed). 


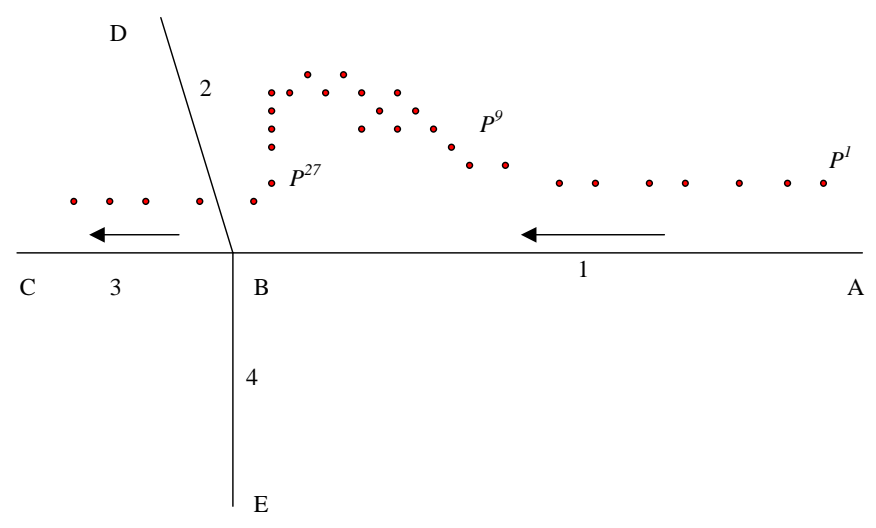

Figure 2: Random GPS/DR points when the vehicle is stationary

Another limitation of existing MM algorithms is associated with the determination of the actual vehicle position on the selected link. Most algorithms adopt a simple perpendicular projection of the position, as established by the positioning sensor, onto the selected link, and ignore the numerous errors associated with the positioning sensors and the spatial road network mapping data. Scott and Drane (1994) looked at this issue and used an optimal map estimate method rather than perpendicular mapping, which is also referred to as the Nearest Point (NP) estimator (see Table 1 for limitations).

To overcome these limitations, a general MM algorithm is proposed in this paper and described in the next section.

\section{DESCRIPTION OF THE MAP MATCHING ALGORITHM}

The MM algorithm analyzed in this paper seeks to correct for some of the deficiencies of other methods as previously described. The basic characteristics of the algorithm include the use of output from the GPS/DR EKF algorithm developed by Zhao et al. (2002), including position, velocity, and time. Information on the vehicle trajectory is used to avoid sudden switching of mapped locations between unconnected road links. The topological aspects of the road network and GPS/DR heading and speed information allow improvements in performance of the algorithm, especially at intersections. In addition the physical location of the vehicle on the selected link is determined from the weighted averages of the two state determinations. The weighting factors are determined empirically, as described further below. Finally, the errors associated with the bearing of the link (due to the map resolution and curvature of the link) and positioning sensors are considered to determine the physical location of the vehicle on the link. The details of 
the algorithm are described below by first describing and defining the data inputs, followed by a description of the map matching process, and a procedure for identifying data outliers.

\subsection{Definition of Data Inputs}

There are several ways to represent digital spatial road network data. The planar model is currently one of the most commonly accepted models because of its high efficiency and low complexity. In the planar model as described by White et al (2000) and Greenfeld (2002), network representation (N) of a street system ( $\overline{\boldsymbol{N}}$ ) consists of a set of curves in $\boldsymbol{R}^{2}$, each of which is called an arc. Each arc $\boldsymbol{A}$, which is a single representation of the road, can be completely described by a fixed series of points $A^{0}, A^{1}, \ldots \ldots \ldots, A^{n A}$, each of which is in $\boldsymbol{R}^{2}$. Those points are divided into nodes and shape points. The endpoints (i.e., $A^{0}$ and $A^{n A}$ ) of the $\operatorname{arc} \boldsymbol{A}$ are referred to as nodes while the other points (i.e., $A^{1}$, $A^{2}, \ldots . A^{n A-1}$ ) are referred to as shape points.

Arcs without shape points are referred to as lines and arcs with shape points are referred to as polylines. Each polyline consists of a series of lines depending on the number of shape points within the arc. Each line is assumed to be piecewise linear. For simplicity, each shape point is assumed to be a node. Each line and node is associated with a set of identifying attributes. The attributes of the node are the $x$-coordinate and $y$-coordinate that identify the spatial location of the node. A node with the same $x$-coordinate and $y$-coordinate has the same identification number. The attributes of each link are determined from the nodes within the link (i.e., start node and end node). Therefore, connectivity information of links within a given network is derived for input to the MM algorithm. The output of the GPS/DR EKF algorithm used as an input to the MM process includes information on coordinates (eastings and northings), speed, heading and the corresponding covariances. The MM process is described in detail below. 


\subsection{Map Matching Process}

The MM process is shown diagrammatically in Figure 3. The three data sources described above are the link and node data and the positioning data. The process is initiated with nodal matching to identify a correct link (among all the links connected with the closest node to the GPS/DR point) and the determinations of the physical location of the GPS/DR point on that link. The next step analyzes whether the next GPS/DR point can be matched to the link identified at the previous step and then determines its physical location on the link. It is vital to carry out the first step carefully and reliably, as there could potentially be many candidate links.

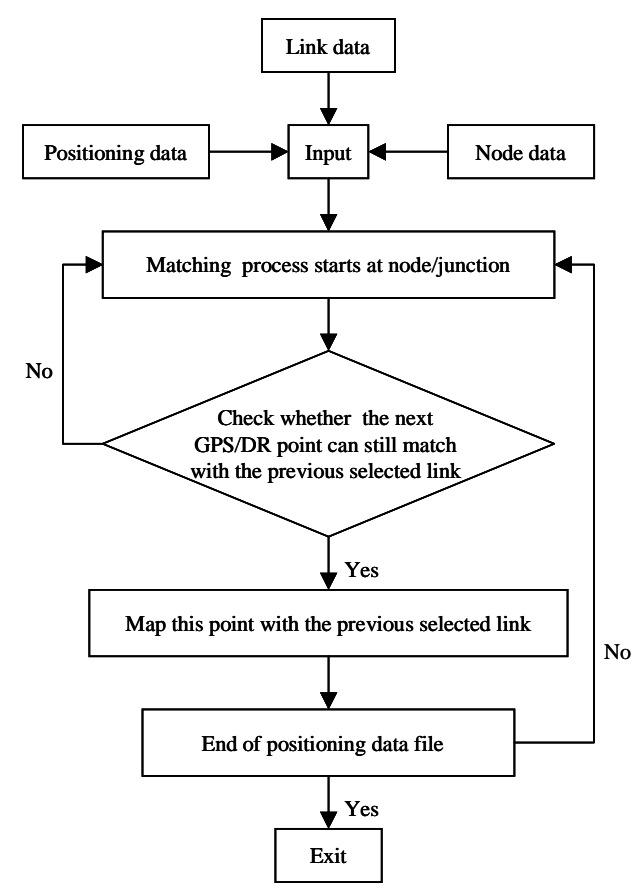

Figure 3: Diagrammatic representation of map-matching algorithm

\subsubsection{Selecting the correct link among candidate links}

The most difficult task of any MM algorithm is to select the correct link among the candidate links. This will occur at junctions in a network. Based on the various similarity criteria between the derived GPS/DR point and the network topology at a junction, a weighting system can be used to select the correct arc as proposed by Greenfeld (2002). The criteria used in the Greenfeld algorithm are the similarity in orientation (i.e., the degree of parallelism between the line formed by two consecutive GPS points and the street network arc), proximity of the point to the street arc and the size of 
the intersecting angle between the GPS derived line and the street arc. The weighting scheme evaluates several candidate arcs for a correct match by computing a likelihood score based on these criteria. Greenfeld (2002) determined that similarity in orientation is more important than proximity, but this can be adjusted based upon the weights assigned to each criteria.

This same procedure is used in the algorithm derived here. To improve the performance of the algorithm, the calibrated DR heading is used instead of the GPS derived line as an input. In addition, the position of the GPS/DR point relative to its closest network node is considered and vehicle speed information is also taken into account. Different weighting factors are used to control for the importance of each of these criteria in determining the best map matching procedure. Each procedure is described in turn in the following sections.

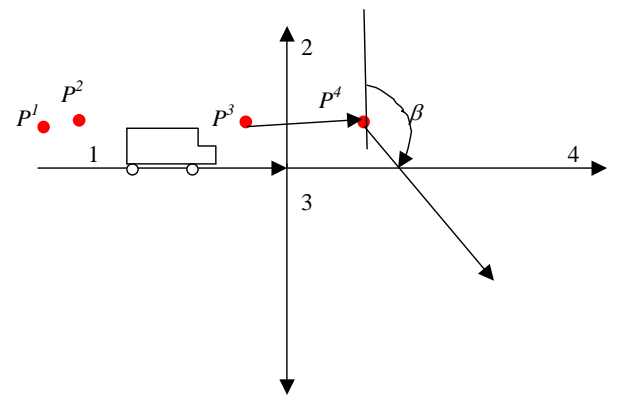

Figure 4: Similarity in vehicle heading and bearing of the link

\subsubsection{Weighting for vehicle heading and bearing of the link}

Suppose the algorithm selected the vehicle position for GPS/DR points $P^{1}, P^{2}$ and $P^{3}$ on link 1 (see Figure 4 ). Now the problem is to identify the next link using information from point $P^{4}$. Since the current position of the vehicle is on link 1, candidate links for point $P^{4}$ are links 2, 3 and 4. To select the correct link, a weighting scheme is applied. The angle $\beta$, provided by GPS/DR, is the heading of the vehicle at point $P^{4}$ measured relative to a northerly direction. This information provides a good indicator of the probable direction of the vehicle at this point. The heading of the link ( $\beta^{\prime}$ ) is then determined from the spatial map data (in this case, $90^{\circ}$ for link $4,0^{\circ}$ for link 2 , and $180^{\circ}$ for link 3 ). The 
difference between the GPS/DR derived heading $(\beta)$ and the spatially derived heading ( $\beta^{\prime}$ ) is $\Delta \beta$ and is used to formulate the weighting scheme as given below.

$$
W S_{H}=A_{H} \cos \left(\Delta \beta^{\prime}\right)
$$

Where,

$$
\begin{aligned}
& \Delta \beta=\beta-\beta^{\prime} \\
& \Delta \beta^{\prime}=\Delta \beta, \text { if }-180^{\circ} \leq \Delta \beta \leq 180^{\circ} \\
& \Delta \beta^{\prime}=360^{0}-\Delta \beta, \text { if } \Delta \beta>180^{0} \\
& \Delta \beta^{\prime}=360^{\circ}+\Delta \beta, \text { if } \Delta \beta<-180^{0}
\end{aligned}
$$

and,

$W S_{H}$ is the weighting score for vehicle heading and orientation of the link

$A_{H}(>0)$ is the weighting parameter for $W S_{H}$ and its value can be obtained from equation (7), described below.

The lower the value of $\Delta \beta^{\prime}$ the higher the probability of the candidate link being the correct link, i.e., as the value of $\Delta \beta^{\prime}$ increases the value of then $W S_{H}$ decreases, as defined by the use of the cosine function. Moreover, the cosine function allows negative weights if $\Delta \beta^{\prime}$ is greater than $90^{\circ}$.

\subsubsection{Weighting for proximity of a point to a link}

Two types of weighting scores are used for the proximity test. The first is based on the perpendicular distance from the GPS/DR point to the link.

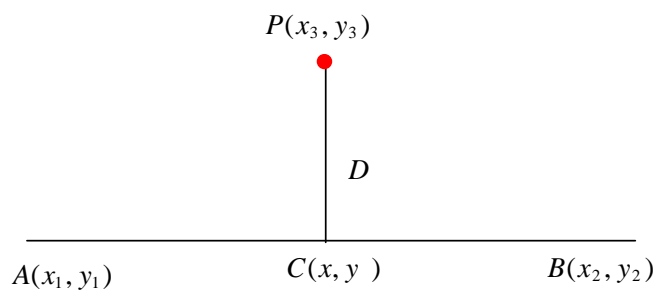

Figure 5: Perpendicular distance 
Suppose $P\left(x_{3}, y_{3}\right)$ is the GPS/DR point and AB is a link (see Fig 5), then the perpendicular distance from this point to the link is

$$
D=\frac{x_{3}\left(y_{1}-y_{2}\right)-y_{3}\left(x_{1}-x_{2}\right)+\left(x_{1} y_{2}-x_{2} y_{1}\right)}{\sqrt{\left(x_{1}-x_{2}\right)^{2}+\left(y_{1}-y_{2}\right)^{2}}}
$$

As perpendicular distance $(D)$ decreases, proximity increases, therefore the weighting score for perpendicular distance is defined as,

$$
W S_{P D}=A_{P} / D
$$

where

$W S_{P D}$ is the score for proximity of the point to the street segment

$D(>0)$ is the perpendicular distance from the GPS/DR point to the road segment

$A_{P}(>0)$ is the weighting parameter that controls the value of $W S_{P D}$. Any value of $A_{P}$ can be taken to determine $W S_{P D}$

The smaller the perpendicular distance the higher the score, i.e., if the point $P$ is close to the link $i$, then there is a greater probability that the link is the correct link

Proximity can also be determined by whether the line between two consecutive GPS/DR points (i.e., $P\left(x_{i-1}, y_{i-1}\right)$ and $\left.P\left(x_{i}, y_{i}\right)\right)$ and link $i$ physically intersect. The proximity between these two lines is measured by their intersecting angle, $\theta$ (acute angle). Smaller intersecting angles lead to closer proximity. If these two lines do not physically intersect, then the score is taken as zero. The weighting score for this proximity is

$$
W S_{P I}=A_{P} \cos (\theta)
$$

where

$W S_{P I}$ is the score for an intersection if an intersection exists 
$A_{P}$ is as previously defined

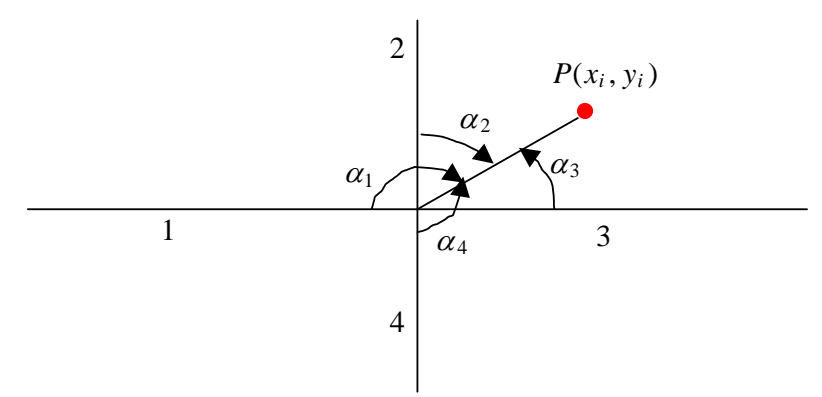

Figure 6: Location of the point relative to the link

\subsubsection{Weighting score for the position of the point relative to the link}

In Figure 6, $P\left(x_{i}, y_{i}\right)$ is a GPS/DR position and the variables $\alpha_{1}, \alpha_{2}, \alpha_{3}$ and $\alpha_{4}$ define the relative location of this point to links 1, 2, 3 and 4 respectively. Since this point can easily map onto either link 2 or link 3, the weighting score must be higher for those links than the other links, i.e., the larger the angle the lower the probability that the link is a correct match. This may not be the case under conditions with multiple paths such as an intersection approach. However, the algorithm also uses weights for the vehicle heading information which would provide the information to deal with these cases. Therefore, the following weighting score of,

$$
W S_{R P}=A_{R P} \cos (\alpha)
$$

where,

$W S_{R P}$ is the weighting score for the position of GPS/DR relative to link

$\alpha(\leq 180)$ is the angle between the candidate link and the line through the nearest node and point

$A_{R P}(>0)$ is the weighting parameter for $W S_{R P}$ and its value can be obtained from equation (7) 


\subsubsection{Total weighting scores (TWS)}

The total weighting score can then be obtained by summing up the individual scores. Therefore, the total weighting score (TWS) is defined as follows:

$$
T W S=W S_{H}+\left(W S_{P D}+W S_{P I}\right)+W S_{R P}
$$

By selecting different values for the weighting parameters, the weight assigned can be carefully controlled. Detailed examinations of field data suggest that the weighting score for heading $\left(W S_{H}\right)$ should be given more importance than that of relative position $\left(W S_{R P}\right)$. One of the reasons is that the heading information used in this study is quite stable as a result of the use of integrated GPS/DR system with an EKF algorithm. Furthermore, the weighting score for relative position ( $\left.W S_{R P}\right)$ should be given more importance than that of proximity ( $\left.W S_{P D}+W S_{P I}\right)$. Therefore, the following formulation is proposed to select the value of the weighting parameters.

$$
\left.\begin{array}{l}
A_{H}=a A_{P} \\
A_{R P}=b A_{P}
\end{array}\right\}
$$

where $a$ and $b$ are the weighting factors and $b>a>1$. The magnitude of $a$ and $b$ depends on the a priori knowledge of the statistical performance of the sensors and the topology of the network. Some preliminary applications of the algorithm with real field GPS/DR positioning data on a representative built-up environment is also necessary to fix the value of $a$ and $b$. After fixing the value of $a$ and $b$, any positive value of $A_{P}$ will provide good results. The value of TWS is then calculated for all the candidate links. The link which provides the highest score is taken as the correct link for that GPS/DR point.

\subsubsection{Weighting results with a typical geometric configuration}

The weighting scheme has been tested using a geometric configuration that could potentially provide an incorrect result. Figure 7 displays a simple geometric configuration with data for a vehicle moving from link 1 to link 3 . As can be seen, there is one data point on link 2, which could indicate that the path followed is from link 1 to link 2. 


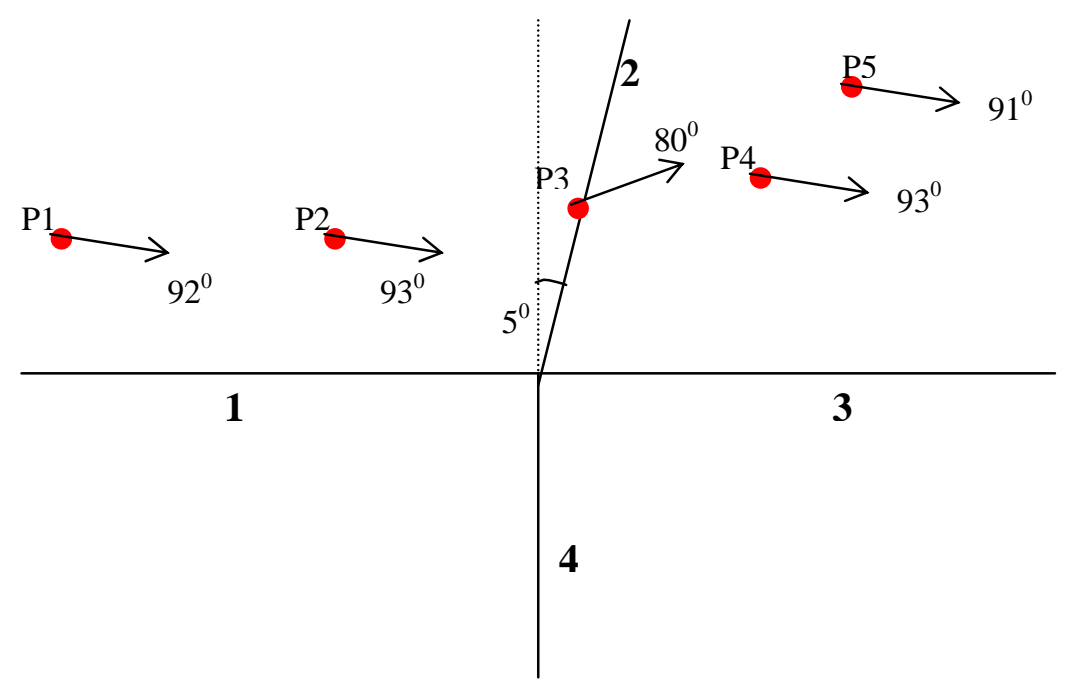

Figure 7: A specific geometric configuration with GPS/DR position fixes

The values along the arrow symbols indicate the vehicle-heading at each position. The identification of the actual link for the points $P^{1}$ and $P^{2}$ using the weighting scheme is very straightforward and it is determined as link 1 . The next step is to identify the link for the point $P^{3}$ which falls on link 2 . One could assume that the weighting scheme gives link 2 the highest TWS for this point since it is exactly on that link. Using the parameters values $a=5, A_{p}=10, b=2$ and the weighting scores derived for each link, Table 2 shows that link 3 has the highest TWS confirming the robustness of the approach.

For further comparison we also applied the weighting scheme as described in Greenfeld (2002) to the above configuration. Using the same parameters as Greenfeld of

$C_{A Z}=10, n_{A Z}=5, C_{D}=10, a=0.1, n_{D}=1.2, C_{I}=10$ and $n_{A Z}=10$, the total score $(W$ ) is calculated as:

$$
W=C_{A Z} \cdot \cos ^{n_{A Z}}(\Delta A Z)+\left(C_{D}-a \cdot D^{n_{D}}\right)+C_{I} \cdot \cos ^{n_{I}}(\Delta A Z)
$$

Results are shown in Table 3. The highest weight is given to link 2 with an indeterminate selection between links 1 and 3, demonstrating the advantages of the method developed here in these types of situations.

Table 2: Calculation of TWS for the point $P^{3}$ using proposed algorithm 


\begin{tabular}{|l|c|c|}
\hline Link & \multicolumn{1}{|c|}{ Variables } & TWS \\
\hline 1 & $\Delta \beta_{1}^{\prime}=10^{0}, D_{1}=40, \theta_{1}=$ nil,$\alpha_{1}=95^{0}$ & 47.74 \\
\hline 2 & $\Delta \beta_{2}^{\prime}=75^{0}, D_{2}=1, \theta_{2}=75^{0}, \alpha_{2}=0^{0}$ & 45.53 \\
\hline 3 & $\Delta \beta_{3}^{\prime}=10^{0}, D_{3}=40, \theta_{3}=n i l, \alpha_{3}=85^{0}$ & 51.23 \\
\hline 4 & $\Delta \beta_{4}^{\prime}=100^{0}, D_{4}=2, \theta_{4}=n i l, \alpha_{4}=175^{0}$ & -23.60 \\
\hline
\end{tabular}

Table 3: Calculation of $W$ for the point $P^{3}$ using Greenfeld's algorithm

\begin{tabular}{|l|l|ll|}
\hline Link & \multicolumn{1}{|c|}{ Variables } & \multicolumn{2}{c|}{ W } \\
\hline 1 & $\Delta A Z=25^{0}, D_{1}=40$ & 7.745 & $\left(C_{I} \approx 0\right)$ \\
\hline 2 & $\Delta A Z=60^{0}, D_{2}=0$ & 10.131 & \\
\hline 3 & $\Delta A Z=25^{0}, D_{3}=40$ & 7.745 & $\left(C_{I} \approx 0\right)$ \\
\hline 4 & $\Delta A Z=155^{0}, D_{4}=2$ & 3.655 & $\left(C_{I} \approx 0\right)$ \\
\hline
\end{tabular}

\subsubsection{Determination of the physical position of the vehicle on the selected link}

The bearing of the link and the vehicle speed $(v)$ from the integrated GPS/DR unit can be used to calculate the vehicle position on a link. Suppose $P^{t}$ and $P^{t+1}$ represent the vehicle position on a link at time $t$ and $t+1$ respectively (Figure 8). The initial easting and northing of the vehicle at point $P^{t}$ is known. From the bearing of the link (i.e., $\theta$ ) and speed of the vehicle at $P^{t+1}$, the increment in easting and northing can be obtained as follows:

$$
\left.\begin{array}{l}
\Delta E_{i}=(v * 1) \sin \theta \\
\Delta N_{i}=(v * 1) \cos \theta
\end{array}\right\}
$$

Therefore, the position of the vehicle at point $P^{t+1}$ can be calculated as follows:

$$
\left.\begin{array}{l}
E_{i+1}=E_{i}+\Delta E_{i} \\
N_{i+1}=N_{i}+\Delta N_{i}
\end{array}\right\}
$$




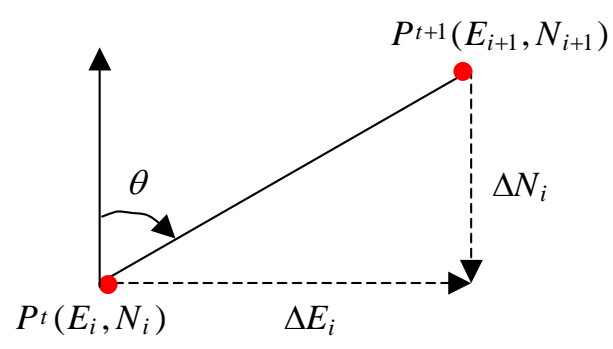

Figure 8: Determination of vehicle position

The other method for determining position is from the GPS/DR positioning sensor (Figure 8). Suppose the GPS/DR unit locates the vehicle at point $P^{S}$ with easting, $E_{S}$ and northing, $N_{S}$. The projected easting ( $P E_{S}$ ) and northing ( $P N_{S}$ ) on the link can be obtained as follows:

$$
\left.\begin{array}{l}
P E_{S}=\frac{\left(x_{2}-x_{1}\right)\left[E_{S}\left(x_{2}-x_{1}\right)+N_{S}\left(y_{2}-y_{1}\right)\right]+\left(y_{2}-y_{1}\right)\left(x_{1} y_{2}-x_{2} y_{1}\right)}{\left(x_{2}-x_{1}\right)^{2}+\left(y_{2}-y_{1}\right)^{2}} \\
P N_{S}=\frac{\left(y_{2}-y_{1}\right)\left[E_{S}\left(x_{2}-x_{1}\right)+N_{S}\left(y_{2}-y_{1}\right)\right]-\left(x_{2}-x_{1}\right)\left(x_{1} y_{2}-x_{2} y_{1}\right)}{\left(x_{2}-x_{1}\right)^{2}+\left(y_{2}-y_{1}\right)^{2}}
\end{array}\right\}
$$

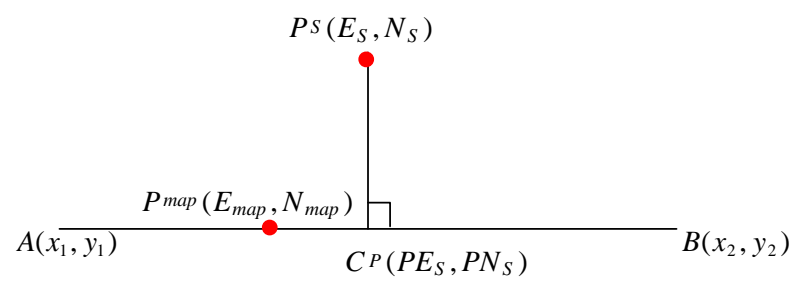

Figure 9: Optimal estimate of vehicle position on the link

Now the two measurements $P^{\text {map }}\left(E_{\text {map }}, N_{\text {map }}\right)$ from the digital map and $C^{P}\left(P E_{S}, P N_{S}\right)$ from sensors are independent (Figure 9). Therefore, the optimal estimation of the vehicle location on the link can be determined as a weighted average of these two measurements as given below. 


$$
\begin{aligned}
& \hat{E}=\left(\frac{{\sigma_{E S}}^{2}}{{\sigma_{E S}{ }^{2}+\sigma_{\text {map }}^{2}}^{2}}\right) E_{\text {map }}+\left(\frac{\sigma_{\text {map }}^{2}}{{\sigma_{E S}{ }^{2}+\sigma_{\text {map }}^{2}}^{2}}\right) P E_{S} \\
& \hat{N}=\left(\frac{\sigma_{N S}^{2}}{{\sigma_{N S}{ }^{2}+{\sigma_{\text {map }}}^{2}}^{2}}\right) N_{\text {map }}+\left(\frac{\sigma_{\text {map }}^{2}}{{\sigma_{N S}^{2}+\sigma_{\text {map }}{ }^{2}}^{2}}\right) P N_{S}
\end{aligned}
$$

where

$$
\begin{aligned}
& \hat{E} \quad \text { is the estimated vehicle position (easting) on the selected link } \\
& \hat{N} \quad \text { is the estimated vehicle position (northing) on the selected link } \\
& \sigma_{\text {map }}{ }^{2} \text { is the variance of errors associated with } E_{\text {map }} \\
& \sigma_{E S}{ }^{2} \text { is the variance of errors associated with } E_{S} \\
& \sigma_{N S}{ }^{2} \text { is the variance of errors associated with } N_{S}
\end{aligned}
$$

As can be seen from equation (9), the error sources associated with the measurement of $E_{\text {map }}$ are those associated with the speed of the vehicle from the GPS/DR unit and the bearing of the link (due to the digital map resolution and curvature of the link). All the variances associated with these errors need to be considered to compute $\sigma_{\text {map }}{ }^{2}$. However, $\sigma_{S}{ }^{2}$ can be obtained directly from the GPS/DR unit.

\subsubsection{Examining whether the vehicle is still on the current link}

After assigning the vehicle position for the first two GPS/DR points on a correct link, the algorithm will examine whether the vehicle is still on the link. Therefore, two conditions have been introduced to test this criterion. They are:

- The difference between the bearings of the two consecutive GPS/DR lines (i.e., lines between $P\left(x_{i}, y_{i}\right)$ and $P\left(x_{i+1}, y_{i+1}\right), P\left(x_{i+1}, y_{i+1}\right)$ and $\left.P\left(x_{i+2}, y_{i+2}\right)\right)$ that is greater than $45^{0}$

- The difference between the heading of the two consecutive GPS/DR points is greater than $45^{\circ}$ and the alpha $(\alpha)$ is greater than $90^{\circ}$ 
If any of the above conditions are true, then the algorithm assumes that the vehicle is approaching an intersection and initializes the map matching process. Otherwise, the vehicle is still on the previous link and the new location is calculated using equation (11).

\subsection{Outlier Identification}

With the integrated GPS/DR sensor, a significant number of outliers can be eliminated using well known quality indicators. However, there is still the possibility of obtaining some outliers or spikes. This could happen at a node or any other position. The point $P\left(x_{i+1}, y_{i+1}\right)$ in Figure 10, is identified as an outlier if $\Delta \delta>45^{0}$ (i.e., if there is a sudden change in two consecutive GPS/DR line bearings). This outlier may cause matching errors especially if $\Delta \delta$ is bigger and closer to arc DF. To avoid such matching errors an outlier is finalized only after the next points have been observed and analyzed. A detailed description on filtering outliers can be found in Greenfeld (2002).

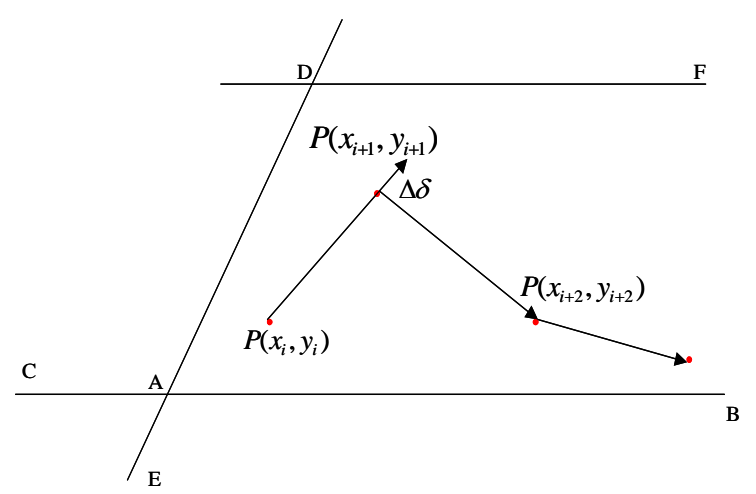

Figure 10: Outlier treatment

\subsection{Algorithm Step by Step}

The detailed description of the map-matching algorithm can be found in Figure 11. The algorithm uses the following steps to assign the vehicle on the correct link and to determine its position on that link.

1. Find the closest node from the first GPS/DR point (i.e., initial point). 
2. Check whether the next point is an outlier. If not, then select all the road segments that pass through the closest node, otherwise take this point as the initial point and go to step-1.

3. Using the weighting formula (Equation 6), choose the correct link. These two points (i.e., initial point and its next point) should be matched to this link.

4. Determine the vehicle position on the correct link for each of the two points using Equation 11.

5. Check whether the next point is an outlier. If yes, then go to step-1 and take it as the initial point. If not, check whether $\Delta \beta^{\prime}<45^{0}$ and $\alpha \leq 90^{\circ}$. If yes, map this point on the same link and determine its position (by equation 11) and continue this process until the above conditions are true, otherwise go to step-1.

6. Repeat step-5 until all points have been matched. 


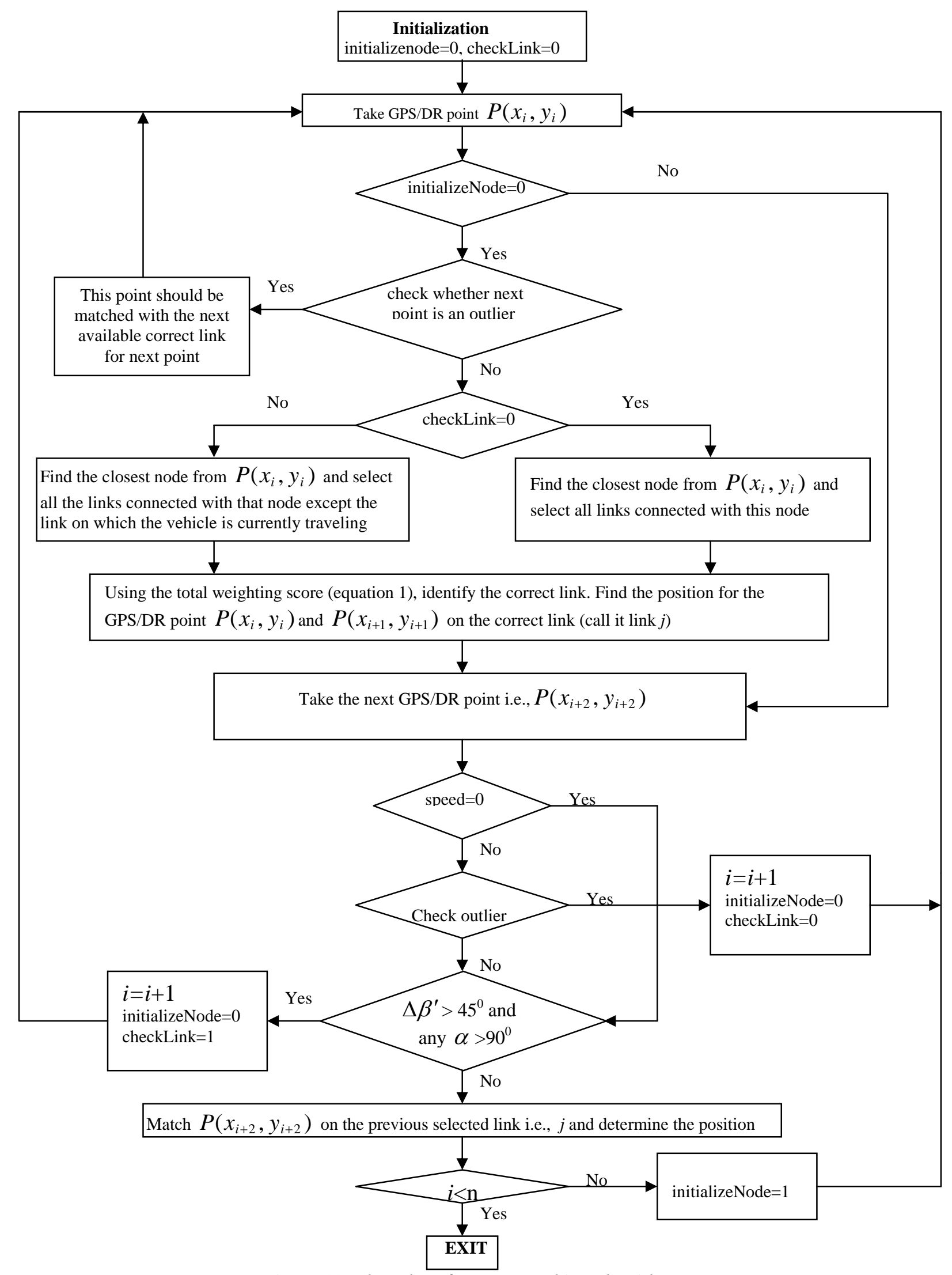

Figure 11: Flow chart for map matching algorithm 


\section{ALGORITHM TESTING}

To test the performance of the MM algorithm, a comprehensive field test was carried out in Central London in January 2002. A test vehicle was equipped with a navigation platform consisting of a 12-channel single frequency GPS receiver, a low-cost MEMS rate gyroscope and the interfaces required to connect to the vehicle speed sensor (odometer) and back-up indicator. The route in London was chosen carefully to have a good mix of important spatial urban characteristics including open spaces, urban canyons, tall buildings, tunnels, bridges, and potential sources of electromagnetic interference. Data capture to sample the temporal variation in the geometry of the GPS satellite constellation was also considered.

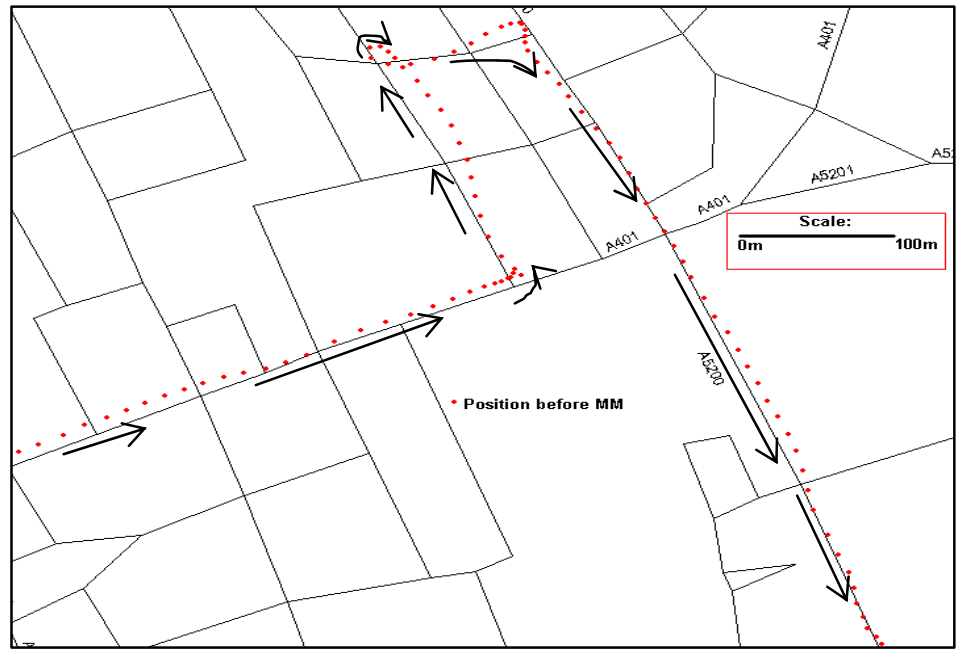

Figure 12: The position measurement from output of GPS/DR EKF algorithm

The positioning results from the output of the integrated (GPS/DR) system based on the EKF algorithm for a sample of the experimental route in Central London are shown in Figures 12 and 13. Each of the dots represents the vehicle position. Note that in most cases the points deviated from the actual route taken due to positioning errors in the integrated system (although 100\% of all position fixes were within 50m of the road center-line). The spatial network data used as reference had a resolution of $1.25 \mathrm{~m}$. The arrow symbols in the figures show the path followed by the vehicle on the network. As already mentioned, the purpose of the MM algorithm developed here is two-fold; first, to match each of the vehicle positions to a link (road segment); and, second, to determine the vehicle location on that link. 


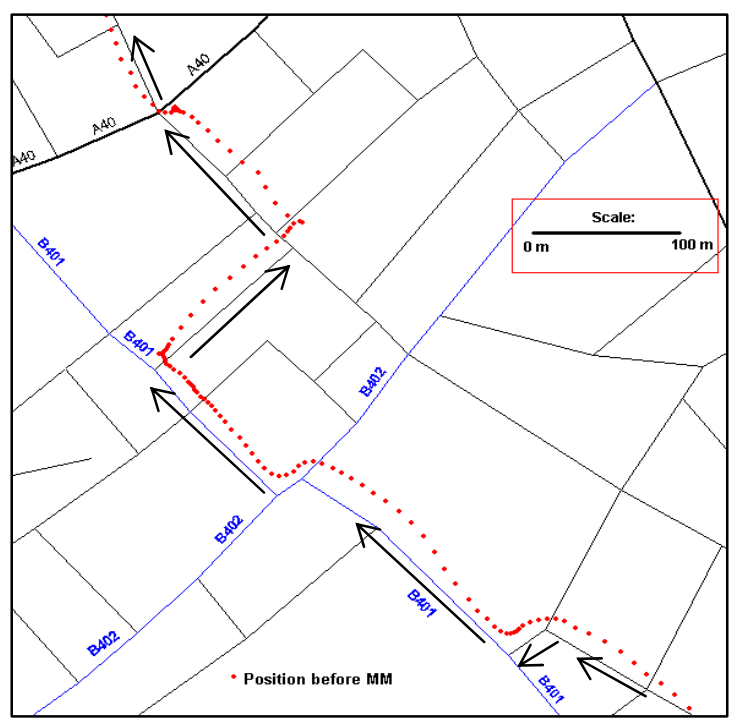

Figure 13: The position measurement from output of GPS/DR EKF algorithm

Figures 14 and 15 illustrate the results of the MM algorithm for the sample routes shown in Figures 12 and 13

respectively. Equation (6) was used to select the actual link among a number of candidate links. After some groundwork application of the algorithm with real field data, the values of the weighting factors $a$ and $b$ are taken as 5 and 2 respectively whereas the value of the weighting parameter $A_{P}$ is taken as 10 . However, any positive value for $A_{P}$ (with $a=5$ and $b=2$ ) was found to provide very good results. The corresponding values of the weighting parameters $A_{H}$ and $A_{R P}$ are 50 and 20 respectively (see Equation-7).

Figure 14 shows what appears to be an aberration in the algorithm in the upper left corner. This was actually a test of the MM algorithm where an intentional U-turn was made. It was found that the algorithm was able to correctly identify the link in this test as well.

In these two cases, almost 100\% correct link identification was achieved. However, it was found that the weighting scheme to identify actual links is sensitive to the weighting factors ( $a$ and $b$ ). More research is therefore needed to obtain optimal values for the weighting parameters. 


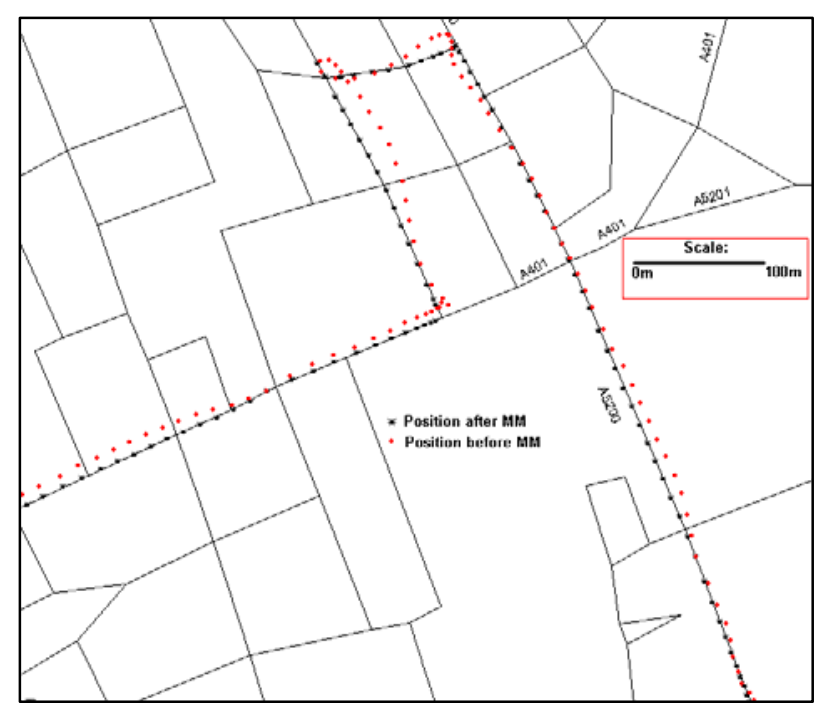

Figure 14: Map matching results corresponding to Fig 12

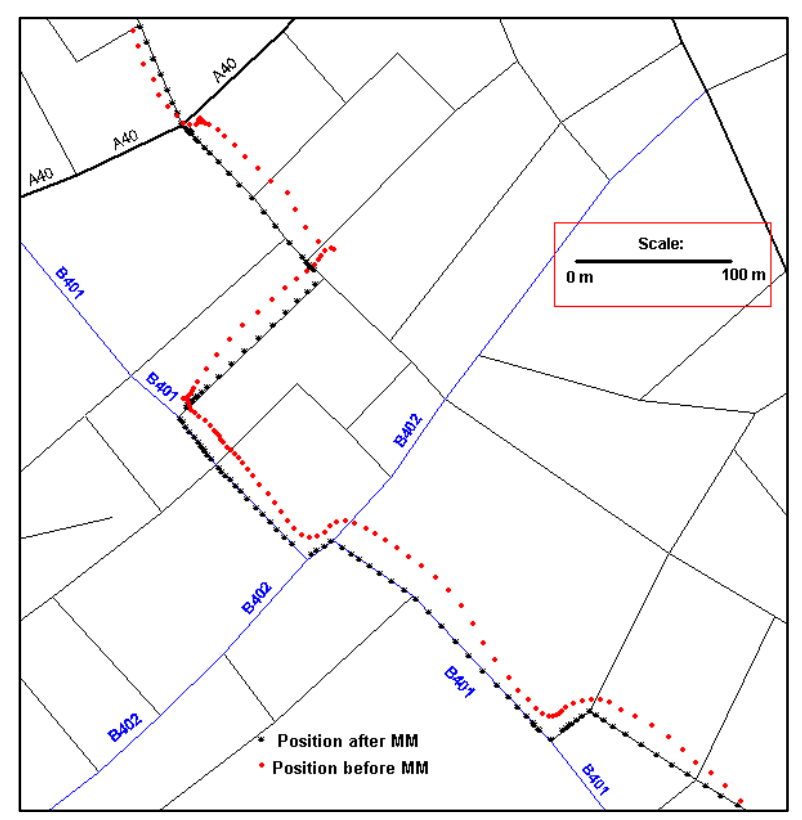

Figure 15: Map matching results corresponding to Fig 13

Equation (11) was then used to determine the actual position of the vehicle on the selected link. A weighted average (optimal estimation) of the two state determination of the vehicle position was used, resulting in robust physical location of the vehicle. Careful observation of the results found that there are sudden losses of data just after some intersections 
in Figure 15. The sensitivity of the weighting factors, as discussed above, and the very short length of the link (e.g., some are below 20m) might be responsible for such clusterings of vehicle location. Research is on going to look at this issue particularly with smaller links in urban networks. Future work will also test the algorithm against the vehicle position as determined from a higher accuracy GPS observable, the carrier phase. The errors associated with map resolution, assumption of linearity of the link, speed of the vehicle and positioning sensors were all taken into account to estimate vehicle position on the link.

\section{CONCLUSIONS}

An integrated system employing GPS and low cost dead reckoning devices, and using an extended Kalman filter has been developed. The resulting vehicle position accuracy is such that map matching is required to accurately locate a vehicle on a given road segment (or link). In this paper, an algorithm based on the topological analysis of the road network utilizing heading and speed information from the EKF algorithm has been developed. The approach adopts a weighted average of two state determinations of the vehicle position based on topological information and external sensors to determine the actual location on a link. The method has been found to be very efficient particular in difficult operational environments such as junctions and intersections. Since the errors associated with the bearing (azimuth) of the link (due to map resolution and the assumption that 'each arc is piece-wise linear') and positioning sensors are taken into account, the estimation of the vehicle position on the selected link is found to be quite robust.

\section{REFERENCES}

Bernstein D., Kornhauser A., 1996. An introduction to map matching for personal navigation assistants. New Jersey TIDE Center (http://www.njtude.org/reports/mapmatchintro.pdf).

Greenfeld, J.S., 2002. Matching GPS observations to locations on a digital map. In proceedings of the $81^{\text {th }}$ Annual Meeting of the Transportation Research Board, Washington D.C.

Jo, T., Haseyamai, M., Kitajima, H., 1996. A map matching method with the innovation of the Kalman filtering. IEICE Trans. Fund. Electron. Comm. Comput. Sci. E79-A, pp 1853-1855. 
Kim, J.S., Lee, J.H., Kang, T.H., Lee, W.Y., Kim, Y.G., 1996. Node based map matching algorithm for car navigation system, Proceeding of the $29^{\text {th }}$ ISATA Symposium, Florence, Vol. 10, pp 121-126.

Krakiwsky, E.J., Harris, C.B., Wong, R.V.C., 1988. A Kalman filter for intrgrating dead reckoning, map matching and GPS positioning. In: Proceddings of IEEE Position Location and Navigation Symposium, 39-46.

Ochieng, W.Y., Sauer, K., 2001. Urban road transport navigation requirements: performance of the global positioning system after selective availability. Transportation Research Part C, 10, 171-187.

Scott, C.A., Drane, C.R., 1994. Increased accuracy of motor vehicle position estimation by utilizing map data, vehicle dynamics and other information sources. In: Proceedings of the Vehicle Navigation and Information Systems Conferences, 585-590.

Tanaka, J., Hirano, K., Itoh, T., Nobuta, H., Tsunoda, S., 1990. Navigation system with map-matching method. Proceeding of the SAE International Congress and Exposition, pp 40-50.

Taylor, G., Blewitt, G., Steup, D., Corbett, S., Car, A., 2001. Road reduction filtering for GPS-GIS navigation.

White, C.E., Bernstein, D., Kornhauser, A.L., 2000. Some map matching algorithms for personal navigation assistants. Transportation Research Part C 8, 91-108.

Zhao, L., Ochieng, W.Y., Quddus, M.A and Noland, R.B., 2002. An Extended Kalman Filter algorithm for Integrating GPS and low-cost Dead reckoning system data for vehicle performance and emissions monitoring. Submitted to the Journal of Navigation. 